\title{
A Comunidade de Pesquisa em Sistemas de Informação no Brasil na perspectiva do Simpósio Brasileiro de Sistemas de Informação
}

\author{
Renata Araujo ${ }^{1}$, Célia Ralha ${ }^{2}$, Alexandre Graeml ${ }^{3}$, Alexandre Cidral ${ }^{4}$ \\ ${ }^{1}$ Programa de Pós-Graduação em Informática - Universidade Federal do Estado do Rio \\ de Janeiro (UNIRIO) - Av. Pasteur, 458, Urca - Rio de Janeiro - RJ - Brasil - 22.290- \\ 240 \\ ${ }^{2}$ Departamento de Ciência da Computação - Universidade de Brasília (UnB) - Caixa \\ Postal 4466 - Brasília - DF - 70.904-970 \\ ${ }^{3}$ Programa de Pós-Graduação em Computação Aplicada - Universidade Tecnológica \\ Federal do Paraná (UTFPR) - Campus Curitiba, Bloco B - $1^{\circ}$ andar. Av. Sete de \\ Setembro 3165, Rebouças - Curitiba - PR- 80.230-901 \\ ${ }^{4}$ Universidade da Região de Joinville (UNIVILLE) Rua Paulo Malschitzki, 10 - Bom \\ Retiro, Joinville - SC - 89219-710 \\ renata.araujo@uniriotec.br; ghedini@cic.unb.br,graeml@utfpr.edu.br, \\ alexandre.cidral@univille.net
}

\begin{abstract}
This paper depicts the history of the Information Systems research community in Brazil over the past 10 years. This portrait is drawn from the perspective of the Brazilian Symposium on Information Systems - a scientific event promoted by the Brazilian Computer Society. The paper discusses what motivates this community, how it has been structured, grown, which research themes the community is interested in, and what are its challenges for the following years.

Resumo. Este artigo apresenta o histórico de 10 anos de atuação da comunidade de pesquisa em Sistemas de Informação no Brasil sob a perspectiva do Simpósio Brasileiro de Sistemas de Informação - evento promovido pela Sociedade Brasileira de Computação. Neste histórico são apresentadas informações sobre o que motiva esta comunidade e como ela tem crescido e se caracterizado em termos de estrutura de pesquisadores $e$ instituições, áreas temáticas de pesquisa e seus principais desafios.
\end{abstract}

\section{Introdução}

A proliferação de cursos de graduação em Sistemas de Informação (SI), o amadurecimento da formação nesta área e o acompanhamento da evolução da pesquisa no contexto internacional levaram a Sociedade Brasileira de Computação (SBC) ${ }^{1}$, por

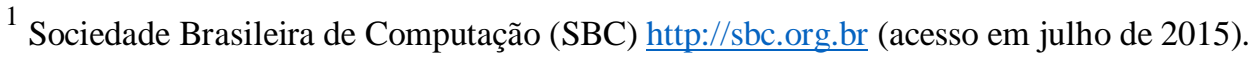


meio de sua Diretoria de Educação e, em particular, do Grupo de Trabalho 2 (GT-2), a definir um Currículo de Referência para os cursos de Bacharelado em SI [SBC, 2013]. Esse currículo tem permitido um direcionamento objetivo da formação de profissionais em SI em âmbito nacional, sendo utilizado como base pelos cursos de graduação no país [MEC, 2012]. Organizações internacionais também têm realizado esforços no sentido de definir o corpo de conhecimento para a formação nas áreas de Ciência da Computação, Engenharia de Software, Engenharia da Computação e Sistemas de Informação. O currículo de referência proposto conjuntamente pela Association for Computing Machinery $(\mathrm{ACM})^{2}$ e a Association for Information Systems (AIS) ${ }^{3}$ para formação superior em SI é abrangente o suficiente para contemplar as particularidades de cursos de departamentos que mais frequentemente se envolvem na oferta desta formação, garantindo um senso de propósito, habilidades e competências esperados dos egressos distintos e distintivos daqueles proporcionados por outros cursos [ACM, 2010]. O que há de comum nestas referências nacionais e internacionais é a visão aplicada de SI, voltada a formar profissionais capazes de compreender os problemas em diferentes amplitudes - sociedade e organizações - sugerindo soluções com uso da tecnologia e processamento de informação.

O grande interesse pela área no Brasil fica ainda evidenciado pelo surgimento e estruturação de distintos grupos de pesquisa nacionais em SI, bem como o estabelecimento de cursos e ações de formação no nível de pós-graduação [Araujo e Barros, 2011]. No entanto, a discussão do que é a pesquisa em SI no país é ainda recente e há necessidade de consolidação. A comunidade internacional tem debatido a natureza desta área de pesquisa [Agarwal e Lucas, 2005][Sidorova, Evangelopoulos Ramakrishnan, 2007][Sidorova et al., 2008]. Além disso, a importância cada vez maior dos SI para o funcionamento das organizações e da sociedade atual justifica o debate sobre diretrizes e uma agenda para a pesquisa em SI em âmbito nacional.

Entende-se que a pesquisa em SI busca compreender a Computação como meio ou instrumento para a solução de problemas do mundo real, da sociedade e das organizações. Seu caráter aplicado gera desafios devido à necessidade de se apropriar de modelos teóricos para a sua consecução, além de modelos de validação prática, que possam ser considerados relevantes e suficientemente rigorosos para sua aplicação [Recker, 2013]. Em seu caráter técnico, a área de SI, em muitas situações, precisa se apropriar de referenciais teórico-práticos de áreas como a Ciência da Computação, Ciência da Informação, a Administração e a Economia, entre outras. Por envolver aspectos não totalmente técnicos, pode se beneficiar ainda de conhecimentos da Psicologia, da Sociologia e demais áreas das Ciências Sociais. Isto é absolutamente necessário para que possa subsidiar aquilo que constitui a sua própria razão de ser: investigar as possibilidades de soluções tecnológicas sistêmicas para problemas complexos no mundo real em diferentes domínios de aplicação [IEEE, 2011, p. 54].

Este artigo pretende contribuir para essa discussão, destacando oportunidades, bem como ações para a consolidação e convergência dos esforços em andamento por parte dos pesquisadores na área. Em particular, nos baseamos na evolução da comunidade de pesquisa em SI no Brasil reunida ao longo de dez anos na realização do Simpósio Brasileiro de Sistemas de Informação (SBSI), associada à SBC.

\footnotetext{
2 http://www.acm.org (acesso em julho de 2015)

3 http://aisnet.org (acesso em julho de 2015)
} 
Historicamente, a área de SI tem sido vista por pesquisadores e educadores da Computação como a intersecção de algumas subáreas específicas mais consolidadas, notadamente as áreas de Engenharia de Software e Banco de Dados, com o viés principal de entender a construção destes sistemas. No entanto, ao se confrontar com os problemas do mundo, uma visão integrada não só destas duas subáreas, mas de várias outras subáreas da Computação (Redes de Computadores, Inteligência Artificial, Algoritmos e Otimização, para citar algumas) tem se tornado inevitável, a fim de compreender o artefato SI aplicado em um contexto específico de demanda e utilização. $\mathrm{O}$ desafio está principalmente em encontrar formas de gerenciar de maneira eficaz a complexidade inerente à pesquisa nesta área, por sua diversidade inerente e exigência de aplicação prática, tornando necessária a compreensão, domínio e utilização de abordagens de pesquisa não usuais para os pesquisadores da área de Computação até então.

Um resumo do histórico desta comunidade foi apresentado durante o X SBSI, em Londrina ${ }^{4}$, no painel "10 anos de SBSI", motivando a escrita deste artigo. O artigo procura analisar, com base no que ocorreu ao longo das diversas edições do evento como esta comunidade tem se caracterizado e o que tem sido apresentado como questões de pesquisa pela área. Discute o que motiva (ou deveria motivar) esta comunidade de pesquisa e observa oportunidades e desafios para que a área de SI se mantenha atuante e gerando resultados relevantes para a pesquisa científica e para o país.

\section{De que comunidade estamos falando?}

No Brasil, embora com diferentes perspectivas, SI têm sido objeto de pesquisa de diferentes domínios - Computação, Administração, Ciências Sociais, Ciência da Informação, Comunicação - para citar algumas. Como reflexo da estrutura políticooperacional do grande sistema de pesquisa brasileiro, a distinção das comunidades científicas em grandes áreas nem sempre facilita a integração de visões e objetivos de pesquisa ao redor de um eixo comum.

Assim, ainda é difícil caracterizar a comunidade de pesquisa em SI de forma global, sob o ponto de vista de todas as áreas que a desenvolvem, mesmo em âmbito nacional. Por esta razão, neste artigo o foco fica restrito, como já mencionado, à comunidade de pesquisa em SI originária da área de Computação, embora, desde sua origem, ocorra alguma interação, ainda que pouco sistemática, com pesquisadores da área de Administração.

As origens da comunidade de SI no âmbito da comunidade de pesquisa em Computação no Brasil datam de 1999, quando, no contexto da SBC, foi estabelecido um grupo de trabalho para discutir as diretrizes curriculares nacionais para os cursos de graduação em Computação e Informática no Brasil. Neste cenário de discussão surge a proposição para a definição dos cursos de Bacharelado em SI.

Nos anos subsequentes (2000-2003), a identidade destes cursos, bem o currículo de referência para a formação na área de SI, foram largamente discutidos, culminando, em 2003, com a aprovação do currículo de referência para os cursos de SI, no âmbito da

\footnotetext{
${ }^{4}$ X Simpósio Brasileiro de Sistemas de Informação - http://www.sbsi2014.uel.br/ (acesso em julho de 2015)
} 
SBC. Ainda em 2003, foi proposta a criação de um evento científico nesta área, dando origem, em 2004, ao primeiro Simpósio Brasileiro de Sistemas de Informação - SBSI, que ocorreu em Porto Alegre (RS).

De 2004 a 2014, o SBSI percorreu nove estados localizados nas cinco regiões do Brasil, dando contorno a uma comunidade de pesquisadores na área, por meio da divulgação de suas comunicações científicas e a discussão de temas desafiadores para SI. Em 2010, a comunidade passou a ter uma referência dentro da estrutura da SBC com a criação da Comissão Especial em Sistemas de Informação (CE-SI) ${ }^{5}$, cujo propósito é definir estratégias e políticas de pesquisa para a comunidade de SI, sendo formada por um grupo de pesquisadores da área, que se renova anualmente.

Dados coletados das edições anuais do SBSI de 2005 a 2014 mostram que a comunidade reunida no evento apresenta regularidade na exposição de suas pesquisas, envolvendo um número significativo de pesquisadores autores investigando as questões da área e comunicando seus resultados (Figura 1). Ressalta-se neste gráfico o fato de que em 2007 o evento não foi realizado e os anais do evento em 2004 não estão disponíveis para o levantamento.

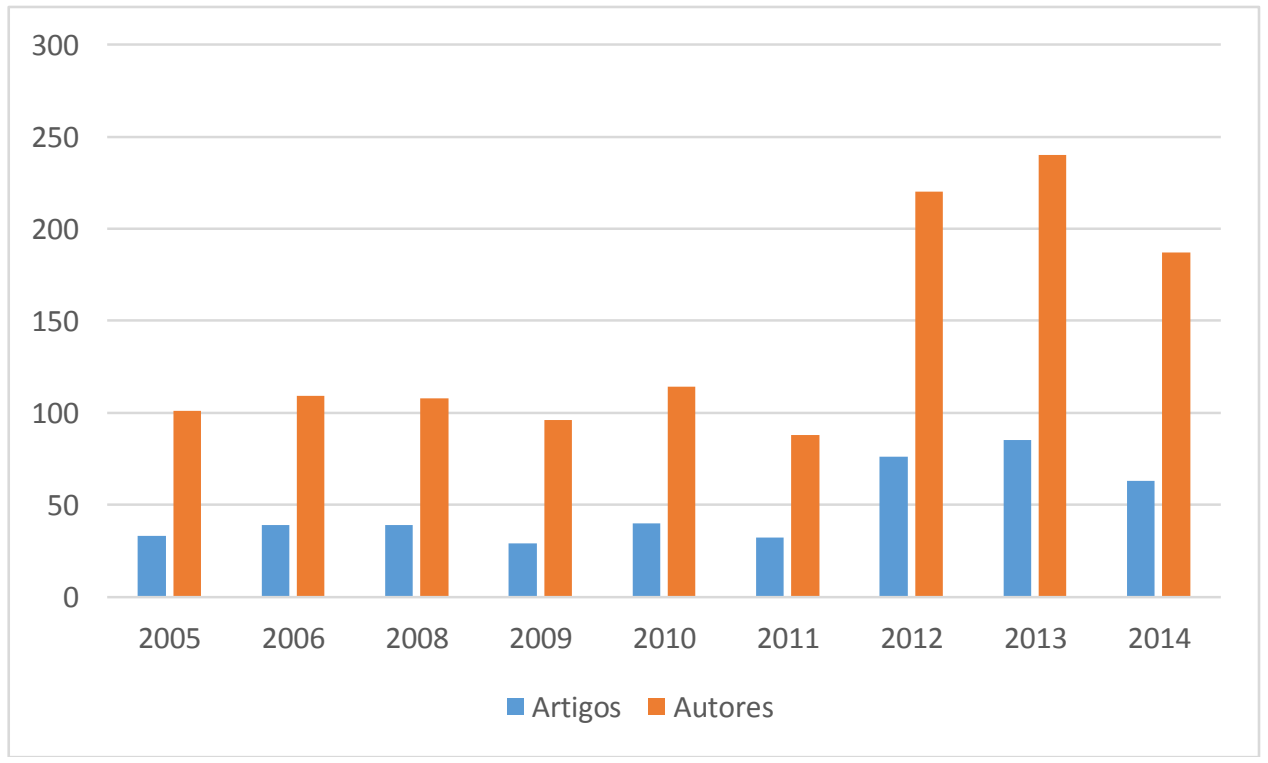

Figura 1 - Total de artigos e autores por edição do SBSI 2005 a $2014^{6}$.

\section{Como esta comunidade de pesquisa tem atuado?}

Com a finalidade de conhecer melhor a comunidade de pesquisa que se reúne no SBSI, foram desenvolvidos por pesquisadores desta comunidade, trabalhos científicos voltados à mineração de dados, à análise de redes sociais e à coleta de informação com base nos anais do evento [Silveira e Muramatsu, 2011][Oliveira, 2012][Rodrigues, 2015][Rodrigues e Ralha, 2015]. Os trabalhos de Rodrigues (2015) e Rodrigues e Ralha (2015), mais amplos, cobriram a realização do SBSI de 2005 a 2014, considerando os anais disponíveis.

\footnotetext{
5 Comissão Especial de Sistemas de Informação. Sociedade Brasileira de Computação. http://sbc.org.br/ce-si/ (acesso em julho de 2015)

${ }^{6}$ Os anais do evento em 2004 não fizeram parte da coleta e o evento não ocorreu em 2007.
} 
Por meio destas pesquisas foi possível observar que a comunidade de SI, ao longo de sua primeira década, pôde nuclear pesquisadores e instituições como referências de pesquisa na área. A Figura 2 apresenta o volume de publicação dos quinze autores com maior frequência de publicação nas edições de 2005 a 2014 do SBSI. A média de publicação destes autores é de aproximadamente um artigo por ano, o que indica a preocupação destes pesquisadores em contribuir e fazer parte desta comunidade regularmente.

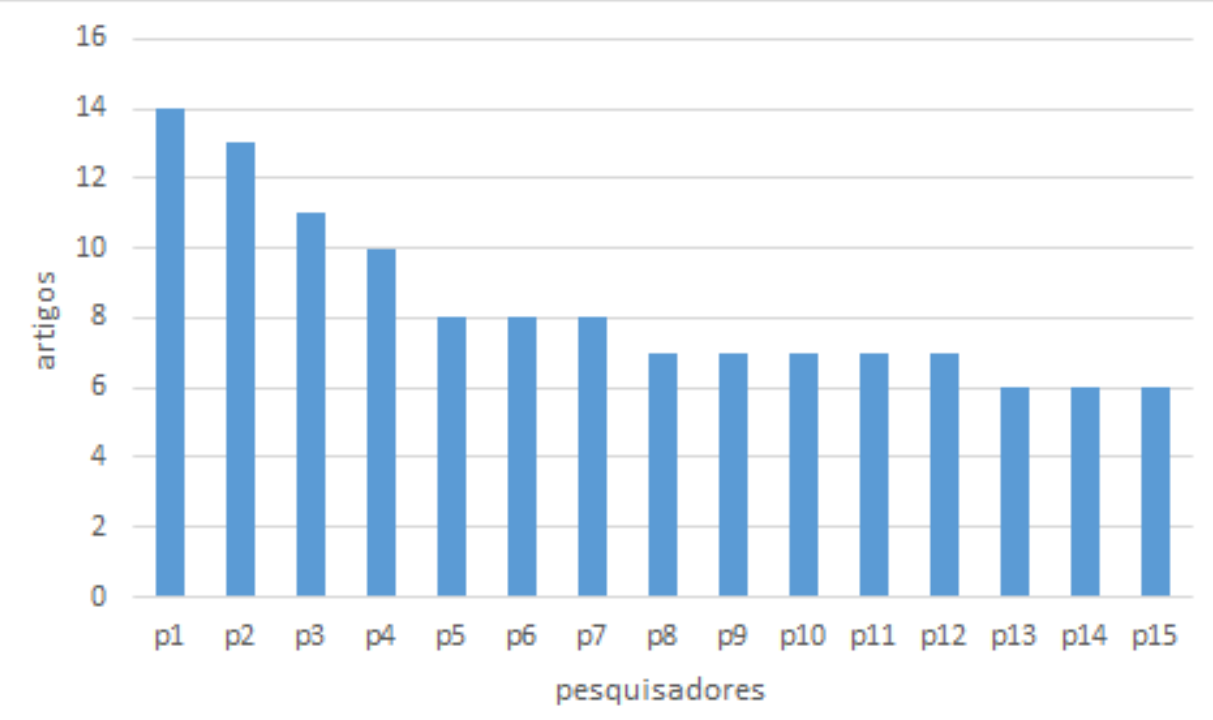

Figura 2: Volume de publicação dos 15 autores com maior frequência de publicação nas edições 2005-2014 do SBSI

A Figura 3 apresenta as quinze instituições de vinculação dos autores com maior frequência de publicação no evento ao longo deste período. Observa-se variedade nas instituições presentes, sobretudo em seu aspecto regional. O quantitativo de publicações por instituição demonstra também o esforço de presença no evento sobretudo de instituições que lideram programas de pós-graduação e pesquisa na área de SI. 
QUANTIDADE DE AUTORES

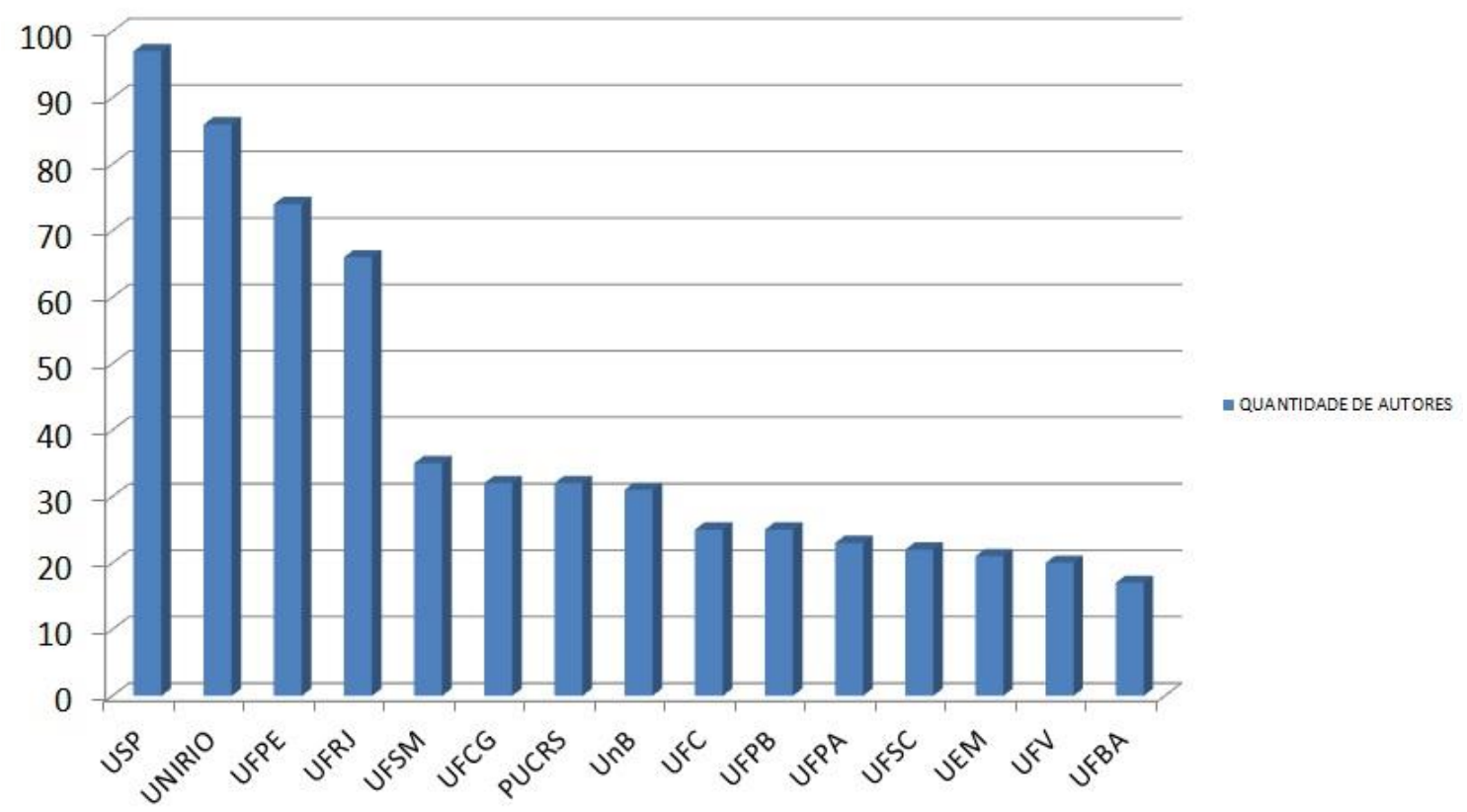

Figura 3: As instituições de vinculação dos autores com maior frequência de publicação nas edições 2005-2014 SBSI

Outras análises demonstram que os pesquisadores com maior frequência de publicação no evento estabelecem redes significativas de coautoria. Por exemplo, na Figura 4 observa-se a rede de co-autoria de um autor (402, nó central) ao longo de suas publicações no evento. Os autores demonstram também continuidade de publicação ao longo do tempo, conforme apresentado na Figura 5.

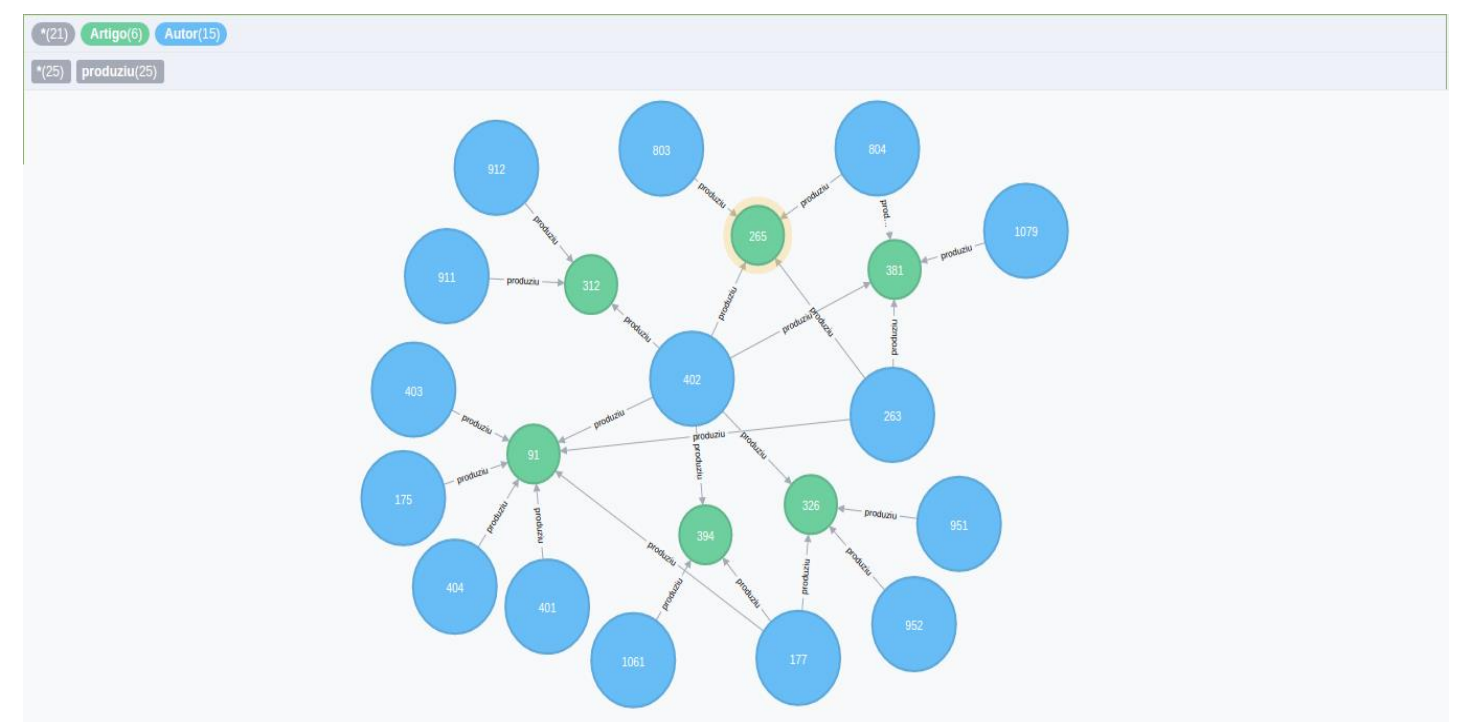

Figura 4: Representação em grafo de coautores de determinado autor 


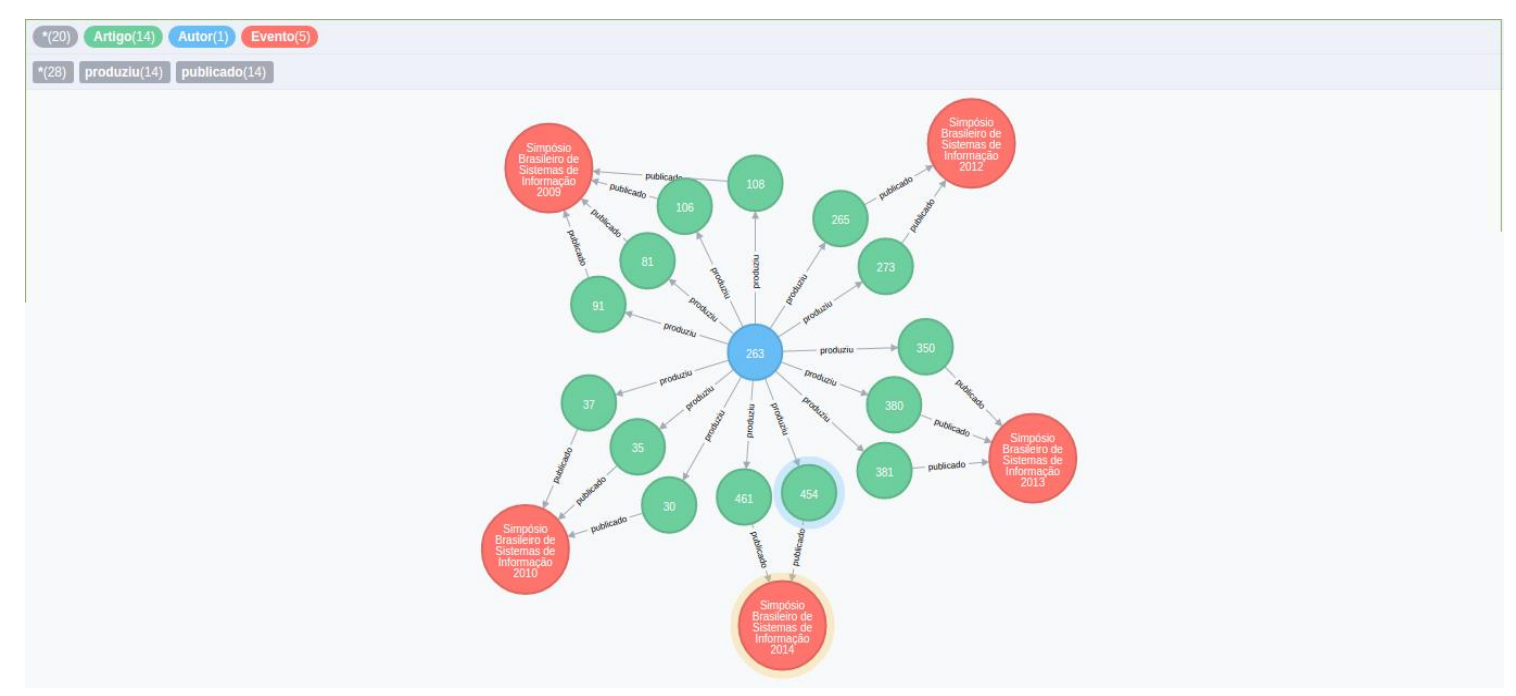

Figura 5: Representação em grafo das publicações de um autor específico nas edições do SBSI

\section{O que motiva esta comunidade?}

É natural que a gênese de uma nova disciplina, ou área de pesquisa, seja muito influenciada por outras disciplinas que lhe sejam próximas. Em assim sendo, não surpreende que SI tenha chamado a atenção, em um primeiro momento, dos pesquisadores da Computação, já que seu objeto prioritário de investigação são tecnologias e sistemas computacionais, e daqueles que começaram a ver suas atividades modificadas pela introdução dos novos artefatos tecnológicos em seu cotidiano, dentre os quais merecem destaque os Administradores.

O SBSI, tendo sido organizado, desde as suas primeiras edições, por uma comunidade científica que se estruturou a partir da área da Computação, tem acolhido trabalhos que, em sua maioria, concentram seu foco na análise do artefato tecnológico, ainda que preocupados com a forma como esses afetam o ambiente em que são inseridos. Eventos patrocinados pela Associação Nacional de Pesquisa e Pós-graduação em Administração ${ }^{7}$, por sua vez, dão maior ênfase às transformações organizacionais e questões associadas ao indivíduo e grupos sociais que fazem parte dos macro-sistemas organizacionais em que estão inseridos os sistemas computacionais dentro de empresas. A partir da perspectiva adotada pelos pesquisadores de SI ligados à área de Administração, o sistema computacional é parte integrante de um sistema maior, que, para autores como Laudon e Laudon (2015) compreendem uma dimensão humana e uma dimensão organizacional, além dos componentes técnicos.

\footnotetext{
7 Associação Nacional de Pós-Graduação e Pesquisa em Administração. http://www.anpad.org.br/ anpad/. (acesso em julho de 2015)
} 
Neste sentido, a partir de 2008, as edições do SBSI foram marcadas por contextos temáticos norteadores de grandes questões de pesquisa para a área que envolvem tanto os aspectos técnicos como as dimensões não técnicas deste artefato, acompanhando as grandes questões nacionais e mundiais nesta área: "Desafios para a pesquisa em SI no Brasil" (2008), "Sistemas de Informação e o Cidadão Brasileiro: Acesso Participativo e Universal à Informação e ao Conhecimento" (2009), "Desafios para o Desenvolvimento Sustentável Apoiado em TI" (2010), “Academia, Governo e Empresa: interação, desenvolvimento e inovação m Sistemas de Informação (2011), "Cidades Inteligentes" (2012), "Sistemas de Informação e os Desafios do Mundo Aberto" (2013), "Ecossistemas Digitais e Estratégicos" (2014), e "Sistemas de Informação: uma visão sociotécnica da Computação" (2015).

Transversalmente a estas grandes questões norteadoras, a comunidade de pesquisa foi compartimentando sub-áreas mais específicas de intervenção. Estas áreas demonstram o posicionamento de interesse desta comunidade em relação a questões de pesquisa mais particulares inseridas nos contextos de: Sistemas de Informação de Governo, Gestão de Processos de Negócio e mais recentemente os aspectos de Ensino e Inovação em SI. Estes temas foram consolidados pela criação de workshops e trilhas específicas no escopo do evento ao longo dos anos.

\section{O que deve continuar motivando a comunidade de SI?}

Os pesquisadores em SI tendem a adotar perspectivas bastante pragmáticas na sua abordagem de suas pesquisas, seja enfatizando o artefato tecnológico ou sua inserção dentro de organizações. Há décadas, contudo, pesquisadores de diversas outras áreas sinalizam com questões fascinantes para discussão em SI, as quais ainda se tornam mais atuais à medida que artefatos computacionais invadem todos os aspectos da vida humana. Quem não se inquieta com a provocação de McLuhan (1964, p. 3) de que: "no longo prazo, o conteúdo transmitido por um meio importa menos que o próprio meio para influenciar a forma como a gente pensa e age"? Se "o meio é a mensagem", aqueles que desenvolvem o meio ou propõem a sua inclusão em ambientes compartilhados com/por humanos têm uma responsabilidade enorme pelo que estão fazendo, não?

Para Wolton (2003, p. 97): “ontem, as coisas eram simples: o que dependia do telefone era diferente do que dependia do rádio e da televisão, e distinto do que concernia ao computador. Os terminais diferentes remetiam a atividades diferentes, a áreas diferentes, a culturas diferentes. Amanhã, ao contrário, tudo estará disponível no mesmo terminal. A mudança não é somente técnica, é também cultural, uma vez que não haverá mais diferenças entre atividades separadas durante séculos." Aquilo que há poucos anos consistia em previsão para o futuro representa, cada vez mais, a realidade não só das organizações, mas dos lares mundo a fora. $O$ "amanhã" de Wolton já se faz presente no dia-a-dia da maior parte das pessoas. Na mesma caixa (ou caixinha, se estivermos falando do telefone celular) são oferecidos recursos e serviços que antes precisavam de diversos dispositivos tecnológicos distintos.

Cabe, portanto, aos pesquisadores de SI desenvolver uma reflexão mais profunda sobre as tecnologias que estudam e seus desdobramentos. Talvez muitos de nós discordem, ao menos em termos mais gerais, da percepção de Postman (1995) de 
que "nossas novas tecnologias são como barganhas de Fausto: sempre nos dão algo, mas ao fazê-lo, também tiram de nós algo importante". Ainda assim, temos que reconhecer que a forma como temos adotado (individual e coletivamente) novas tecnologias demonstra pouca ou nenhuma preocupação com seus eventuais "efeitos colaterais" ou simples consequências da forma como nos apropriamos de novas tecnologias.

Estudar os desdobramentos de nossas escolhas tecnológicas sobre o indivíduo e sobre a sociedade envolverá, cada vez mais, o suporte de disciplinas das Ciências Sociais, como já mencionado anteriormente. Pesquisadores em SI precisarão se preocupar com a questão, trazendo da Psicologia, da Sociologia, da Comunicação e de qualquer outra área que possa proporcionar subsídios para tal discussão, as ferramentas necessárias para compreender e fazer compreender nosso mundo tecnológico, principalmente agora que os SI avançaram das organizações produtivas para dentro da casa das pessoas e a vida de qualquer cidadão desse planeta.

Postman $(1998,1999)$ propõe seis questões, cuja análise considera que possa nos ajudar a compreender como e porque SI vem sendo implantados, além dos seus impactos sobre indivíduos e a sociedade. São elas: 1) Qual é o problema para o qual a tecnologia em questão se propõe a ser a solução? 2) De quem é esse problema? 3) Que novos problemas podem ser criados ao se resolver o problema original? 4) Que pessoas e instituições são mais seriamente afetadas pela nova tecnologia? 5) Que mudanças na língua são causadas pela nova tecnologia? 6) Que pessoas e instituições ganham poder econômico e político com a adoção da nova tecnologia?

$\mathrm{Na}$ visão do autor, as seis questões enunciadas acima podem ser motivo de reflexão individual, para serem aplicadas à própria vida da pessoa, mas, se consideradas de forma mais abrangente, envolvendo toda a sociedade, podem assumir uma dimensão política maior [Postman, 1998]. Na verdade, é quase impossível pensarmos nelas apenas no âmbito pessoal, uma vez que "vivemos em rede" e as decisões de cada um de nós afetam os demais. Segundo McLuhan (1962, p. 1), "a tecnologia eletrônica [...] torna o individualismo obsoleto e [...] a interdependência entre organizações, obrigatória". Não é mais possível pensar sozinho, ou fazer nada sozinho.

A colaboração se torna imprescindível em um mundo mediado por redes e o impacto disso sobre o indivíduo, sobre as organizações e sobre a sociedade não pode ser ignorado, justificando nosso convite para que nossa comunidade se debruce com mais afinco sobre as consequências dos sistemas que desenvolvemos e estudamos. Temos nos concentrado demasiadamente nos meios e dado pouca atenção aos fins, conforme já percebia o poeta norte-americano Henry David Thoreau, lembrado por Postman (1994), para quem temos uma clara propensão a nos transformarmos em "ferramentas de nossas próprias ferramentas" (p. 13), as quais "nada mais são do que meios aperfeiçoados para se chegar a um fim não melhorado" (p. 16).

\section{Quais os desafios para sua evolução?}

Em 2009, a comunidade de pesquisa reunida no SBSI elaborou uma agenda de ações para os dez anos subsequentes [Cidral et al., 2009]. Nesta agenda, cinco desafios principais foram apontados para a área, a saber: i) a definição de referencial teórico para a pesquisa em SI, que possa caracterizar suas principais questões e permitir flexibilidade para aplicação em domínios diversos e de constante evolução, de acordo com os 
avanços tecnológicos e sociais; ii) a abertura à multi/inter/transdisciplinaridade, caracterizando níveis de relevância e rigor necessários à pesquisa desta natureza; iii) o desenvolvimento de competências em diferentes metodologias de pesquisa, tanto quantitativas quanto qualitativas, mas com ênfase no rigor e relevância da sua aplicação, para a obtenção de contribuições científicas efetivas para a área e para a sociedade em geral; iv) o exercício contínuo de pesquisa aplicada aos problemas da indústria, do governo ou da sociedade, de forma realmente integrada e conjunta; e v) a caracterização do perfil profissional do pós-graduado em Sistemas de Informação, alinhada às demandas da indústria e do mercado.

Estes desafios evidenciam a preocupação da área desde então em atender às provocações de Poston e Wolton mencionadas na seção anterior. Tratam de posicionar a comunidade de pesquisa quanto à necessidade de alterar suas concepções e estratégias de pesquisa, bem como capacitar esta comunidade à utilização de métodos que alcancem os objetivos de uma pesquisa aplicada de alto nível. Dada a natureza dos problemas atuais e a escala com a qual a tecnologia se torna presente em todas as atividades humanas, não há mais espaço para desenvolver pesquisa relevante nesta área sem que uma abordagem sociotécnica seja utilizada [Albuquerque, 2015]. Para uma comunidade formada por egressos de pesquisa em uma área fim, como a Computação, esta mudança de perspectiva não é trivial e precisa ser trabalhada fortemente não só na comunidade atual, como também no âmbito da formação de novos mestres, doutores e pesquisadores que comporão a área no futuro.

Considerando o perfil, a constituição e o histórico desta comunidade, é importante pontuar a necessidade de mudar a estratégia de caracterização da área. Uma perspectiva para esta mudança pode ser deixar em segundo plano a definição do que compreende ou não o escopo da pesquisa em SI em termos de temas ou assuntos de pesquisa para uma visão que possibilite à comunidade identificar nichos de problemas e questões de pesquisa relevantes. Ou seja, o que caracteriza a pesquisa em SI não são assuntos específicos, mas cenários problema reais e relevantes das organizações e da sociedade atual. Alia-se a esta questão a necessidade de caracterização de cenários problema específicos para o contexto nacional, envolvendo as particularidades da realidade das organizações e sociedade brasileiras.

Tendo em vista seu caráter aplicado, a comunidade de pesquisa em SI precisa também desenvolver novas alternativas de disseminação de seus resultados. Isto porque as implicações de sua pesquisa impactam diretamente o cotidiano de pessoas e organizações. A relevância e impacto da pesquisa na área de SI na sociedade e organizações requerem uma dinâmica de discussão de seus resultados que envolva mais do que a comunidade acadêmica, mas as organizações e a sociedade em si. Alternativas como a ciência aberta (Open Science) ${ }^{8}$ ou a ciência participativa (Citizen Science) ${ }^{9}$ precisam ser exploradas neste contexto.

\section{Conclusão}

Este artigo marca os dez primeiros anos de atuação da comunidade de pesquisa em Sistemas de Informação nucleada pelo SBSI. É inquestionável a existência desta

\footnotetext{
${ }^{8}$ Open Science. https://en.wikipedia.org/wiki/Open_science (acesso em julho de 2015)

${ }^{9}$ Citizen Science. https://en.wikipedia.org/wiki/Citizen_science (acesso em julho de 2015)
} 
comunidade, haja vista a continuidade na participação de pesquisadores e instituições, bem como sua atuação estratégica junto a associações científicas como a SBC e AIS.

A pesquisa em SI tem sido de interesse de diversas áreas científicas, sobretudo no que se refere à sua aplicação e impactos. No âmbito da comunidade de pesquisa que se reúne no SBSI, uma mudança de visão da natureza da pesquisa desenvolvida é ainda necessária. A apropriação de alternativas que ampliem suas metodologias de pesquisa para o estudo de cenários problema complexos e multidisciplinares é aspecto chave para a consolidação e fortalecimento da área.

\section{Referências}

ACM (2010), The Curriculum Guidelines for Undergraduate Degree Programs in Information Systems, Association for Computing Machinery and Association for Information Systems. https://www.acm.org/education/curricula-recommendations (acesso em julho de 2015).

AGARWAL, R., LUCAS, H.C. (2005), The Information Systems Identity Crisis: Focusing on High-Visibility and High-Impact Research. MIS Quarterly. vol. 29, n. 3, pp. 381-398.

ALBUQUERQUE, J. P. (2015), Sistemas de Informação no Brasil em uma Abordagem Sociotécnica. Palestra. Simpósio Brasileiro de Sistemas de Informação 2015. http://www.portal.inf.ufg.br/sbsi2015/node/54. (acesso em julho de 2015)

ARAUJO, R. M., BARROS, M. (2011), Information Systems Graduate Education and Research in Brazil. Revista Eletrônica de Sistemas de Informação, v. 9.

CIDRAL, A., RAlHA, C., CÁCERES, E., SANTORO, F., AUDY, J., BARROS, M., FURTADO, O., ARAUJO, R. (2009), Sistemas de Informação no Brasil - Desafios e Oportunidades. http://sbc.org.br/ce-si/documentosImportantes.html. (acesso em julho de 2015).

IEEE. Nomenclatura Padrão do IEEE para Cursos da Área de Computação na América Latina. 2011.

LAUDON, K. C.; LAUDON, J. P. (2015), Management Information Systems: Managing the Digital Firm. 15. ed. Prentice Hall.

McLUHAN, M. (1964), Os meios de comunicação como extensões do homem (Understanding Media). São Paulo: Cultrix.

MEC (2012), Diretrizes Curriculares Nacionais para os cursos de graduação em Computação, Ministério da Educação. http://portal.mec.gov.br/index.php?option=com content\&id=12991\&Itemid=866 (acesso julho de 2815)

OLIVEIRA, E. A. (2012) Sobre a Colaboração na Comunidade de SI Através dos Simpósios SBSI. Dissertação de Mestrado em Informática, Departamento de Informática Aplicada, Universidade Federal do Estado do Rio de Janeiro (Unirio), Rio de Janeiro/RJ.

POSTMAN, N. (1994), Tecnopólio: a rendição da cultura à tecnologia. São Paulo: Nobel. 
POSTMAN, N. (1995), Technology's Faustian Bargain [registro de vídeo]. PBS's NewsHour.http://www.patheos.com/blogs/godandthemachine/2012/03/technologysfaustian-bargain/. (acesso em fevereiro de 2015).

POSTMAN, N. (1998), Technology and Society [registro de vídeo]. Speech at Calvin College.https://www.youtube.com/watch?v=uglSCuG31P4. (acesso em fevereiro de 2015).

POSTMAN, N. (1999), Building a bridge to the 18th century: how the past can improve our future. New York : Alfred A. Knopf.

RECKER, J. (2013), Scientific Research in Information Systems, Springer, 1a. edição.

RODRIGUES, N. S. (2015a) Análise da Comunidade Brasileira de Sistemas de Informação Utilizando Diferentes Abordagens de Banco de Dados. Monografia em Computação-Licenciatura, Departamento de Ciência da Computação, Universidade de Brasília (UnB), Brasília/DF.

RODRIGUES, N. S.; RALHA, C. G. (2015) Conhecendo a Comunidade de Sistemas de Informação no Brasil: um Estudo Comparativo Utilizando Diferentes Abordagens de Banco de Dados. Em Anais do Simpósio Brasileiro de Sistemas de Informação (SBSI'2015), 26 a 29 maio 2015, Goiânia/GO.

SBC (2013), Currículo de Referência para os cursos de Sistemas de Informação, Sociedade Brasileira de Computação

http://www.sbc.org.br/index.php?option=com_jdownloads\&Itemid=0\&task=view.down load\&catid=36\&cid=185 (acesso em julho de 2015)

SIDOROVA, A. EVANGELOPOULOS, N., RAMAKRISHNAN, T. (2007) Diversity in IS Research: An Exploratory Study Using Latent Semantics. ICIS Proceedings. Paper 10 .

SIDOROVA, A., EVANGELOPOULOS, N., VALACICH, J.S., RAMAKRISHNAN, T., (2008) Uncovreing the Intellectual Core of the Information Sytems Discipline. MIS Quarterly. vol. 32, n.3, pp. 467-482.

SILVEIRA, B. A.; MURAMATSU, T. Y. (2011) Análise do Perfil de uma Comunidade Científica Através de Mineração de Texto. Monografia em Informática, Departamento de Informática Aplicada, Universidade Federal do Estado do Rio de Janeiro (Unirio), Rio de Janeiro/RJ.

WOLTON, D. (2003), Internet e depois? Uma teoria crítica das novas mídias. Porto Alegre: Sulina. 